\title{
A REMARK ON DECOMPOSITIONS OF THE PERMUTATION REPRESENTATION OF A PERMUTATION GROUP
}

\author{
TOSIRO TSUZUKU
}

To Richard Brauer on the occasion of his 60th birthday

Let $\left(\mathbb{S}\right.$ be a permutation group on $n$-letters $1,2, \ldots, n$. Let $\mathbb{S}_{1}$ be the subgroup of $\$$ fixing suitable one letter, say 1 . For any element $G$ of $\mathbb{B}$, a non-singular matrix $G^{*}=\left(g_{i j}\right)$ of degree $n$ is defined by the equation

$$
\left(\begin{array}{c}
1^{G} \\
\cdot \\
\cdot \\
\cdot \\
\underline{n}^{G}
\end{array}\right)=G^{*}\left(\begin{array}{c}
\underline{1} \\
\cdot \\
\cdot \\
\underline{n}
\end{array}\right) .
$$

Since $g_{i j}^{\prime} s$ are 0 or 1 , we may assume that $G^{*}$ is a matrix whose coefficients are in an arbitrary unitary ring $K$. Then if for any element $G$ of $\&$ we take the mapping $G \rightarrow G^{*}$, this mapping will be a representation $P_{K}$ of $\mathbb{B}$ by the nonsingular $n \times n$ matrices over $K$. By the formula (1) the representation $P_{K}$ is also the representation of $\mathbb{B}$ induced by the identity representation of $\mathbb{B}_{1}$ over $K$. We call $P_{K}$ the permutation representation of $\mathbb{G}$ over $K$. If $K$ is a field of characteristic 0 (more generally, if the characteristic of $K$ does not divide the order of $(B)$, then it is well known that $B$ is a doubly transitive group when and only when $P_{K}$ is directly decomposed into two irreducible constituents (see [2]). Now in the present note we consider decompositions of the permutation representation $P_{K}$ of $B$ over an arbitrary unitary ring $K$, instead of such a field of characteristic 0 .

TheOREM 1. Assume that (S) is a doubly transitive group.

i) Ij $n$ is an inversible element of $K$ (e.g. $K$ is a field whose characteristic does not divide $n$ ), then $P_{K}$ is directly decomposed into two indecomposable

Received September 5, 1962. 
constituents and one of them is the identity representation.

ii) If $n$ is not an inversible element of $K$ (e.g. $K$ is rational integer ring or a field whose characteristic divides $n$ ), then $P_{K}$ is a indecomposable representation.

Proof. Let $M_{K}$ be the representation module of $\&$ corresponding to $P_{K}$. Then we may suppose that $M_{K}$ has a basis $1, \underline{2}, \ldots, n$ over $K$ and, for any element $G$ of $\left(3, G^{*}\right.$ operates on this basis such that $i \rightarrow i^{G}$. If $M_{K}$ is directly decomposed into a certain number of $\$$-submodules of $M_{K}$, say $M_{1}, \ldots, M_{r}$, then we have, for $u \in M$ uniquely, $u=\sum_{i=1}^{r} u_{i}, u_{i} \in M_{i}$ and the mappings $\delta_{i}$ : $u \rightarrow u_{i}, i=1, \ldots, n$, are idempotent (3)-endomorphisms of $M_{K}$ such that

$$
\delta_{i} \delta_{j}=0 \quad \text { for } i \neq j \text { and } \sum_{i=1}^{r} \delta_{i}=\text { identity. }
$$

Conversely, if there exist idempotent $\mathbb{B}$-endomorphisms $\delta_{1}, \ldots, \delta_{r}$ of $M_{K}$ satisfying the relations (2), then it is easy to see that $M_{K}$ is directly decomposed into $r$ (3)-submodules of $M_{K}$. Therefore, in order to determine the direct decomposition of $M_{K}$, we need only to look for idempotent (\$-endomorphisms of $M_{K}$ satisfying the relations (2). Let $\delta$ be a $\left(\$-\right.$-endomorphism of $M_{K}$ and put $\underline{i}^{\delta}=\sum_{j=1}^{n} \lambda_{i j} \underline{j}$. Since $\underline{i}^{\delta G}=\underline{i}^{\text {(fo } \delta}$ for any element $G$ of $\mathbb{\&}$, we have $\sum_{j=1}^{r} \lambda_{i j} \underline{j}^{G}=\sum \lambda_{i} G_{\jmath} \jmath$, hence $\lambda_{i j}=\lambda_{i G_{j} G}$ for any element $G$ of $\&$ and for any integers $1 \leqq i, j \leqq n$. Since $B$ is doubly transitive it is easy to see that $\lambda_{11}=\cdots=\lambda_{n n}(=\lambda)$ and $\lambda_{i j}=\lambda_{k_{1}}(=\not k)$ if $i \neq j$ and $k \neq 1$. Hence we have

$$
\left(\begin{array}{c}
1^{\delta} \\
\cdot \\
\cdot \\
\cdot \\
n^{\delta}
\end{array}\right)=\Delta(\delta)\left(\begin{array}{c}
1 \\
\cdot \\
\cdot \\
\cdot \\
n
\end{array}\right) \text { where } \Delta(\delta)=\left(\begin{array}{cccccc}
\lambda & \mu & \cdot & \cdot & \cdot & \mu \\
\mu & \cdot & \cdot & & & \cdot \\
\cdot & \cdot & \cdot & \cdot & \cdot \\
\cdot & & \cdot & \cdot & \cdot & \cdot \\
\cdot & & & \cdot & \cdot & \mu \\
\mu & \cdot & \cdot & \cdot & \mu & \lambda
\end{array}\right)
$$

If $\delta$ is a idempotent 8 -endomorphism of $M_{K}$, i.e. $\delta^{2}=\delta$, then

$$
\Delta ! \delta)=\Delta(\delta)^{2}=\left(\begin{array}{cc}
\lambda^{2}+(n-1) \mu^{2} & \\
\cdot & 2 \lambda \mu+(n-2) \mu^{2} \\
2 \lambda \mu+(n-2) \mu^{2} & \cdot \\
& \lambda^{2}+(n-1) \mu^{2}
\end{array}\right),
$$

therefore we have the equations $\lambda=\lambda^{2}+(n-1) \mu^{2}, \mu=2 \lambda \mu+(n-2) \mu^{2}$. From these equations we see that $\lambda=\mu=0, \lambda=\mu$ and $\lambda n=1, \lambda=1$ and $\mu=0$, or $\lambda=\mu+1$ and $n \lambda=n-1$. Hence if $n$ is not a inversible element of $K$ then we 
have no non trivial idempotent (\$3-endomorphisms of $M_{K}$ and if $n$ is a inversible element of $K$ then there exist exactly two non trivial $\$$-endomorphisms $\delta_{1}, \delta_{\text {. }}$ of $M_{K}$ where

$$
\Delta\left(\delta_{1}\right)=\left(\begin{array}{lll}
\frac{1}{n} & & \frac{1}{n} \\
& \cdot & \\
\frac{1}{n} & \cdot & \frac{1}{n}
\end{array}\right) \text { and } \Delta\left(\delta_{2}\right)=\left(\begin{array}{ccc}
\frac{n-1}{n} & & -\frac{1}{n} \\
-\frac{1}{n} & & \cdot \\
& & \frac{n-1}{n}
\end{array}\right) .
$$

It is easy to see that $\delta_{1} \delta_{2}=\delta_{2} \delta_{1}=0 \delta_{1}+\delta_{2}=$ identity and $M_{K}^{\delta}=k \sum_{i=1}^{n} i$. The proof is complete.

It seems to us of interest to determine irreducible constituents of $M_{K}$. When $B$ is the symmetic groups, H. K. Farahat determined irreducible constituents of $M_{K}$ (see [1]). Using Farahat's method we can prove a following theorem.

TheоRем 2. Let \& be a triply transitive group. If $K$ is a field whose characteristic $p$ divides $n$ and does not divide the order of $\mathbb{G}_{1}$ then, for the (5)-module $M_{K}$, we have a composition series $M_{K} \supset M_{1} \supset M_{2} \supset 0$ where $\operatorname{dim}_{K} M_{2}$ $=\operatorname{dim}_{K} M_{K} / M_{1}=1$.

Proof. Put $M_{1}=\sum_{i=2}^{n} K(i-1)$ and $M_{2}=K \sum_{i=1}^{n} \underline{i}$. Then $M_{1}, M_{2}$ are (S)-submodules of $M_{K}$ and, by our assumption $p \mid n, M_{1} \supset M_{2}$ and $\operatorname{dim}_{K} M_{2}=\operatorname{dim}_{K} M_{K} / M_{1}=1$. Put $M_{1}^{*}=\sum_{i=2}^{n} K \underline{i}$. Then, since $\underline{1}^{G}=1$ for any element $G$ of $\mathscr{G}_{1}$, we see that $M_{1}^{*}$ is a $\mathbb{S}_{1}$-module and there is a $\mathbb{S}_{1}$-isomorphism $\theta$ of $M_{1}$ onto $M_{1}^{*}$, for which $\theta(i-1)=i$. Furthermore, since $n \cdot 1=0$ in $K, \theta$ carries $M_{2}$ onto $M_{2}^{*}=K \sum_{i=2}^{n} i$. It follows that $\theta$ induces a $\mathbb{S}_{1}$-isomorphism of the factor module $M_{1} / M_{2}$ onto the factor module $M_{1}^{*} / M_{2}^{*}$. Since $p$ does not divide the order of $\mathbb{B}_{i}$ and $\mathbb{B}_{1}$ is doubly transitive, $M_{1}{ }^{*} / M_{2}{ }^{*}$ is a irreducible $\mathbb{S}_{1}$-module. It follows that $M_{1} / M_{2}$ is a irreducible $\left(\mathbb{S}_{1}\right.$-module. Hence $M_{1} / M_{2}$ is a irreducible $\mathbb{S}$-module. The proof is complete.

\section{REFERENCES}

[1] H. K. Farahat, On the natural representation of the symmetric groups, Proc. Glasgow Math, Assoc. 5 (1962), 121-136. 
[2] G. Frobenius, Über die Charaktere der mehrfach transitiven Gruppe, Sitzungsber, Preuss. Akad. (1904), 558-571.

\section{Mathematical Institute}

Nagoya University 